\title{
Cutaneous Lymphoproliferative Disorder
}

National Cancer Institute

\section{Source}

National Cancer Institute. Cutaneous Lymphoproliferative Disorder. NCI Thesaurus. Code C7614.

A lymphoproliferative disorder that affects the skin. 